\title{
Stagnation of Steroid Hormones in Patients with Atopic Dermatitis and Unique Variation of Leukocyte Pattern during the Withdrawal Syndrome af- ter Cessation of Steroid Ointment
}

\author{
Minoru FuKUDA', Nobuaki KaWADA ${ }^{2}$, Nobuyo $\mathrm{KATOH}^{3}$, Hiroki KaWAMURA ${ }^{4}$ and Toru $\mathrm{ABO}^{4}$ \\ ${ }^{1}$ Fukuda-iin, Niigata, ${ }^{2}$ Department of Obstetrics and Gynecology, Uhrin Hospital, Fukushima, ${ }^{3}$ Department of Public Health, Juntendo \\ University School of Medicine, Tokyo, and ${ }^{4}$ Department of Immunology, Niigata University School of Medicine, Niigata, Japan \\ (Accepted 20 April 2003)
}

\begin{abstract}
Patients with severe atopic dermatitis are sometimes seen even after 15-20 years of age, despite the fact that this is age when atopic dermatitis subsides in usual cases. We consider the possibility that steroid hormones administered as ointment stagnate in the skin and become oxidized cholesterols. Such substances may induce circulation failure and granulocyte-associated inflammation. We herein report the withdrawal of the above ointment in such patients, resulting in successful treatment within several months. Since they suffered from the withdrawal syndrome for the first two or three weeks, acupuncture was performed. Before therapy, these patients showed elevated levels of granulocytes and eosinophils, and an inverse decreased level of lymphocytes in the blood. The number and proportion of granulocytes are known to increase by sympathetic nerve stimulation. This, therefore, indicated that these patients were in a dominant state of the sympathetic nervous system. During the therapy, this leukocyte pattern became much worse due to the withdrawal syndrome. However, in parallel with the amelioration of inflammation, normalization of the leukocyte pattern was observed. These results revealed that, by some yet-undetermined reasons, patients with atopic dermatitis who had been treated by steroid ointment for long time, showed an unusual pattern of leukocytes (i.e., granulocytosis) and that this leukocyte pattern was normalized by the withdrawal of steroid hormones in parallel with the amelioration of the disease.
\end{abstract}

The onset of atopic dermatitis usually begins at infancy or childhood and some cases experience spontaneous cure before adolescence $(3,12)$. Primarily, people in childhood show a predominant state of the immune system (i.e., a high level of lymphocytes) (5). However, adults gradually show a decreased level of the immune system (9). The level of granulocytes surpasses that of lymphocytes at 15-20 years old in Japan (our unpublished observation). We speculate that this age-associated change of the

Correspondence to: Dr. T. Abo, Department of Immunology, Niigata University School of Medicine, Asahimachi-dori 1, Niigata 951-8510, Japan

Fax: + 81-25-227-0766

E-mail: immunol2@med.niigata-u.ac.jp immune system might be responsible for the spontaneous cure of atopic dermatitis. In other words, many cases of atopic dermatitis naturally subside due to an age-dependent decrease in the number of $\mathrm{T}$ cells.

In spite of the usual course described above, some patients with atopic dermatitis do not show spontaneous cure, but rather suffer from severe symptoms such as inflamed reddish facial skin $(3,12)$. Such patients have a long history of using topical ointment containing steroid hormones. Are these severe cases of atopic dermatitis inevitable because of their atopic nature or curable by some appropriate therapy? We speculate that if steroid hormones contained in ointment remain long in the dermal tissue of the skin, they become oxidized cholesterols. Ster- 
oid hormones have a cholesterol structure, and the skin of patients with atopic dermatitis who use ointment containing such hormones smells bad. This would seem to indicate that the oxidized substance stimulates tissues and infiltration by granulocytes occurs. We confirmed this phenomenon in an animal model (7). Oxidized cholesterols directly induce the accumulation of granulocytes at the corresponding sites (our unpublished observation). Such granulocytes are further activated by many inflammatory cytokines, (e.g., TNF $\alpha$, IFN $\gamma$, G-CSF, etc.), as well as by sympathetic nerve stimulation after the interaction with resident bacteria.

In the past four years, we have attempted to eliminate such oxidized substances from the skin of patients with severe atopic dermatitis and have been successful in almost all such cases (>95\%). Since our patients suffered from the withdrawal syndrome of steroid hormones during therapy, we applied acupuncture. If we knew the actual drug adverse reaction of steroid hormones, we, as well as other clinicians, would be able to successfully treat many patients with severe atopic dermatitis, even those addicted to steroid hormones. Although the precise reasons are not yet determined, granulocytosis seen in patients with severe atopic dermatitis and in the withdrawal syndrome during therapy is interesting. We have to consider the function of granulocytes as well as the functions of IgE, T cells, and eosinophils $(1,20)$, at least in severe cases of atopic dermatitis.

\section{MATERIALS AND METHODS}

Patients. Patients with severe atopic dermatitis (total $\mathrm{n}=89$, male $\mathrm{n}=45$, and female $\mathrm{n}=44$; ages 12 to 28 , average $19.9 \pm 6.0$ ) were treated. The severity of the atopic dermatitis was estimated according to a previously reported system of grading (13). As indicated by the authors in that paper (13) and others (2), the measurement of disease activity in atopic dermatitis is not so easy. If we apply the simplest method to categorize such patients, "mild", "moderate" and "severe" atopic dermatitis groups can be classified (13). Since almost all patients (approximately $100 \%$ ) in this study had used ointment containing steroid hormones for several years, at least $80 \%$ of patients with atopic dermatitis should be classified as "severe" cases. Age-matched healthy controls $(n=100)$ were also selected so as to compare their leukocyte pattern with that of the patients.

Leukocyte pattern. The number of white blood cells (total leukocytes) was enumerated in the blood of healthy controls and patients with severe atopic dermatitis. The proportions of granulocytes (including neutrophils and basophils), eosinophils, and lymphocytes were determined by May-Grünwald-Giemsa staining. These data were obtained for the blood of the controls and that of the patients during the therapy.

Measurement of free catecholamines, vanillylmandelic acid (VMA), 17-ketosteroids (17-KS), and 17$\mathrm{OH}$ corticosteroids (17-OHCS) in urine. Urine was collected from 13 patients with atopic dermatitis (before therapy) and 20 age-matched healthy controls for $24 \mathrm{hrs}$, and the concentrations (/liter) of these substances were measured by the HPLC method (8).

Measurement of serum levels of lipids and cholesterols. All parameters was measured in the laboratory of SRL (Tokyo, Japan, http://www.SRL-inc.co.jp/).

Actupuncture. In addition to psychological support for the patients, we applied acupuncture. Briefly, fingers and toes were punctured with 26-gauge needles; more precisely, sites lateral to the nails were punctured, resulting in some bleeding. This stimulation induced parasympathetic nerve activation, e.g., recovery of peripheral circulation, a decrease in the blood pressure, relief from the itching, etc. In some cases, the severe withdrawal syndrome occurred during the therapy and some elevation of serum transaminases, indicating hepatic failure, was seen. In such severe cases (less than $10 \%$ of the patients treated), transfusion of physiological saline $(500 \mathrm{ml}$ containing a diuretic) ivas intravenously administered once a day (up to several times).

Statistical analysis. Differences between the results obtained from healthy control subjects and patients with atopic dermatitis were analyzed by using Student's $t$-test.

\section{RESULTS}

\section{Immmoparameters in patients}

To determine the immunologic states in patients with severe atopic dermatitis $(n=89)$, the number of leukocytes and the percentage of the leukocyte population were enumerated in the peripheral blood (Table 1). These patients suffered from severe atopic dermatitis of the face and other sites of the body. All patients had used ointment containing steroid hormones for several months to several years. Before visiting our hospital, they had been treated at several 
Table 1 A comparison of the distribution of leukocytes between healthy subjects and patients with atopic dermatitis

\begin{tabular}{|c|c|c|c|c|}
\hline \multirow[t]{2}{*}{ Parameter } & \multirow{2}{*}{$\begin{array}{l}\text { Healthy subjects } \\
\qquad(\mathrm{n}=100)\end{array}$} & \multicolumn{3}{|c|}{$\begin{array}{l}\text { Patients with severe atopic dermatitis } \\
\qquad(\mathrm{n}=89)\end{array}$} \\
\hline & & Before therapy & 2 wks after therapy & At discharge \\
\hline Number of leukocytes & $6,500 \pm 1,180$ & $7,700 \pm 1,800^{*}$ & $8,500 \pm 2,400^{*}$ & $7,500 \pm 1,800^{*}$ \\
\hline$\%$ Granulocytes ${ }^{2}$ & $57.0 \pm 9.2$ & $59.5 \pm 10.7$ & $63.3 \pm 10.9^{*}$ & $52.0 \pm 10.3$ \\
\hline$\%$ Eosinophils & $1.8 \pm 1.0$ & $11.8 \pm 8.4^{*}$ & $13.1 \pm 8.1^{*}$ & $13.7 \pm 8.5^{*}$ \\
\hline \% Lymphocytes & $38.9 \pm 5.2$ & $27.8 \pm 8.6^{*}$ & $22.6 \pm 8.8^{*}$ & $34.2 \pm 9.4$ \\
\hline
\end{tabular}

${ }^{\mathrm{a}}$ Neurophils and basophils (not including eosinophils)

$* \mathrm{p}<0.05$

other hospitals. For a comparison of the leukocyte pattern, age-matched healthy subjects $(n=100)$ were examined in parallel. Before our therapy, these patients showed elevated levels of the number of leukocytes and the proportion of eosinophils, and a decreased level of the proportion of lymphocytes $(\mathrm{P}<0.05)$. As for the absolute number of leukocytes, it was estimated that the numbers of granulocytes and eosinophils, but not of lymphocytes, increased $(\mathrm{P}<0.05)$.

\section{Urine levels of catecholamines}

We previously reported that predominance of the sympathetic nervous system induces an elevation of granulocytes in the peripheral blood and other tissues of humans $(5,8,15)$ and mice $(4,10,22)$. Since this is the case, it was speculated that the sympathetic nervous system of patients with severe atopic dermatitis who showed elevated levels of granulocytes might be predominant. This was obvious from the fact that the majority of the patients suffered from tachycardia and insomnia. We also examined the urine level of catecholamines as well as of metabolites of innate steroid hormones (Fig. 1). The patients showed elevated levels (per day) of free catecholamines and VMA (a metabolite of catecholamines) $(\mathrm{P}<0.05)$. The level of $17-\mathrm{KS}$ was low $(\mathrm{P}<0.05)$ while that of $17-\mathrm{OHCS}$ remained normal $(\mathrm{P}>0.05)$.

\section{Serum levels of lipids and cholesterols}

To examine the possibility that sympathetic nerve activation was induced by oxidized cholesterols derived from stagnated steroids, we measured the serum levels of lipids and cholesterols (Fig. 2). Among tested parameters, the levels of total lipid, total cholesterol, $\beta$-lipoprotein, cholesterol ester, and free cholesterol were comparable between controls and the patients. However, the level of lipid peroxide (which is known to include oxidized cholesterols) increased but that of total bile acid decreased in the patients $(P<0.01)$.

\section{Recovery from atopic dermatitis}

According to our hypothesis (i.e., accumulation of oxidized cholesterols in the skin), we began the treatment of patients. It included 1) the cessation of ointment usage, 2) acupuncture, and 3) psychological support. Two weeks after commencement of the therapy, the leukocyte pattern (the increase in the number of granulocytes and eosinophils) seen in patients with severe atopic dermatitis became much more prominent (see Table 1). Namely, one of the major signs of the withdrawal syndrome was the worsening of the leukocyte pattern, as well as the worsening of inflammation. However, these symptoms gradually subsided (Table 1 and Fig. 3). At the time of discharge from the hospital (Table 1, right column), the leukocyte pattern had become almost normal, except for the value of eosinophils. As shown in Fig. 3, we were able to achieve a remarkable improvement of severe atopic dermatitis in all patients. Time intervals before and after the present treatment ranged from one month to five months. However, at the beginning of therapy, some patients (approximately 5\%) gave up the therapy, dropping out due to the severe withdrawal syndrome. Some patients (approximately 20\%) showed a sporadic exacerbation of atopic dermatitis even after discharge. However, these symptoms gradually disappeared as a function of time. We recommended exercise for these patients since appropriate exercise seems to be very effective for eliminating the exacerbation. 

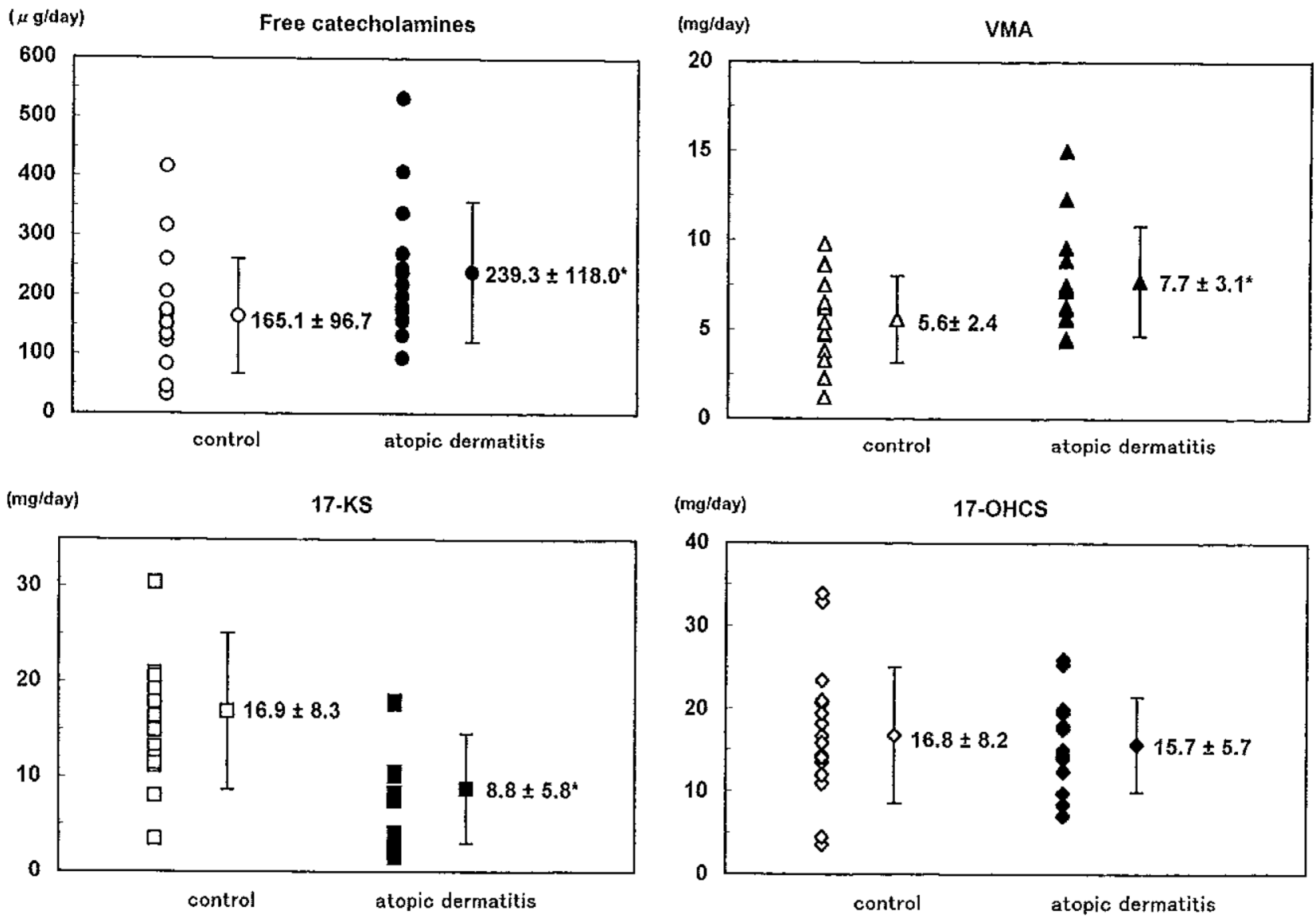

Fig. 1 Excretion of free catecholamines, VMA, 17-KS, and 17-OHCS into the urine (per day) in controls and the patients. Urine was collected from patients with severe atopic dermatitis $(n=13)$ and age-matched healthy controls $(\mathrm{n}=20){ }^{*} \mathrm{P}<0.05$

\section{DISCUSSION}

In a series of recent studies, we reported that immunologic states are under the regulation of the autonomic nervous system $(4,5,8,10,15,17,22)$. This is due to the fact that granulocytes carry surface adrenergic receptors (11) and lymphocytes carry surface cholinergic receptors (17). If the sympathetic nervous system is stimulated, granulocytes are activated in number and function. Inversely, if the parasympathetic nervous system is stimulated, lymphocytes such as $T$ and $B$ cells are activated in number and function. A rapid change in the distribution of leukocytes arises from the fact that the life span of leukocytes is very short, e.g., the life span of granulocytes being only 2 days after maturation (10). Moreover, sympathetic nerve stimulation induces the acceleration of granulocyte trafficking, i.e., the bone marrow (the pool organ of granulocytes $) \rightarrow$ the circulation $\rightarrow$ the mucosal/cutaneous tissues. As a consequence, sympathetic nervous system stimulation increases the number of granulocytes in the blood. This includes the granulocytosis seen in the daytime (the circadian rhythm) (15), that resulting from physical stress (10), and neonatal granulocytosis induced by commencement of pulmonary respiration at birth (5).

In light of these findings, we herein analyzed the leukocyte pattern in patients with severe atopic dermatitis, who had been diagnosed as suffering from an incurable disease (or state) at other hospitals. In contrast to our anticipation, the level of granulocytes was found to be elevated while that of lymphocytes was low in these patients. Namely, they were in an immunosuppressive state. The immunosuppressive state seen in patients with severe atopic dermatitis has been similarly reported by other investigators $(6$, $14,18,19)$.

We postulated that some oxidized substances (e.g., oxidized cholesterols resulting from steroid 

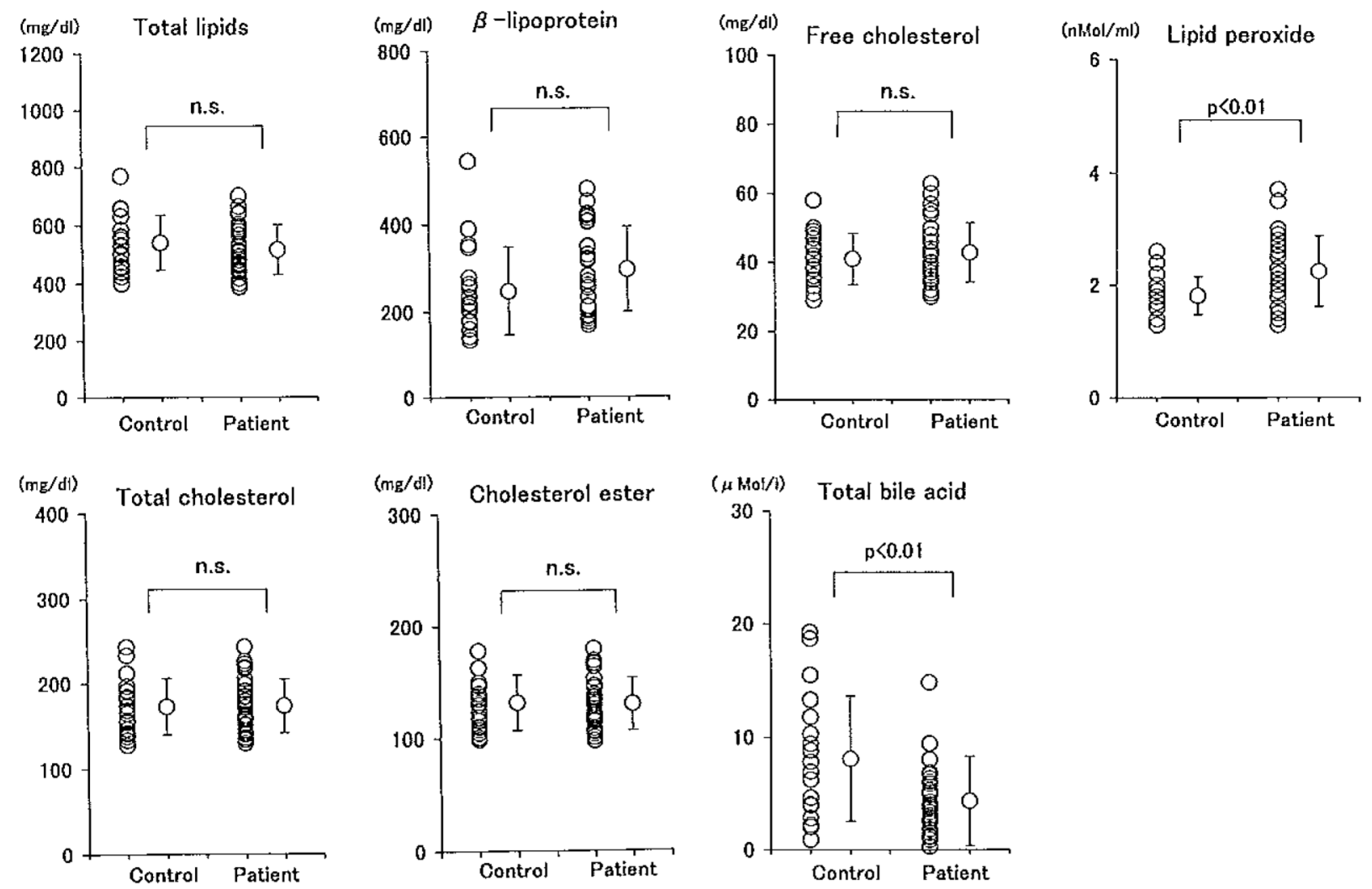

Fig. 2 A comparison of the serum levels of lipids and cholesterols between control and patients with severe atopic dermatitis. Blood samples were collected from severe atopic dermatitis $(n=29)$ and age-matched healthy controls $(n=19)$ and the serum levels of lipids and cholesterols were measured. The level of lipid peroxide increased but that of total bile acid decreased in the patients $(\mathrm{P}<0.01)$.

hormones used as ointment) had induced sympathetic nervous system dominance (Fig. 4). Indeed, almost all of these young patients had symptoms such as tachycardia, hypertension, insomnia, anxiety, fatigue, etc. This postulation was confirmed by the results of urine analysis, namely, the levels of free catecholamines and VMA were elevated in the urine of the patients. We do not know why the level of $17-\mathrm{KS}$ was low in these patients. The elevated serum level of lipid peroxide (which includes oxidized cholesterols) was also found to be elevated in the patients. At present, we do not know why the patients showed a decreased serum level of total bile acid. As mentioned below, one possibility is acceleration of the excretion of oxidized cholesterols as bile acid in the patients.

According to our hypothesis, we discontinued the use of the ointment containing steroid hormones and waited for the oxidized cholesterols to be excreted from the skin. As well established, the usual routes for the excretion of metabolized steroid hormones are the urine (as 17-OHCS) and the bile (as bile acid because of high oxidization). However, if they remain in the tissue for long, it is speculated that they become oxidized cholesterols which then induce granulocyte-associated inflammation. Finally, they may induce atherosclerosis and aging, as seen in some patients, if not all, with asthma and autoimmune diseases such as scleroderma, who have used steroid hormones for a long time (i.e., several years or longer).

Our successful treatment of severe atopic dermatitis supports our hypothesis. During the therapy, patients experienced the excretion of a purulent substance from the skin. To overcome the withdrawal syndrome from steroid hormones, we applied acupuncture as well as giving psychological support. However, when we applied acupuncture without the cessation of steroid hormones, the effects were limited or nil (our unpublished observation). Acupuncture induces the reflex of parasympathetic nerve stimulation $(11,16,21)$ and results in the recovery 
a

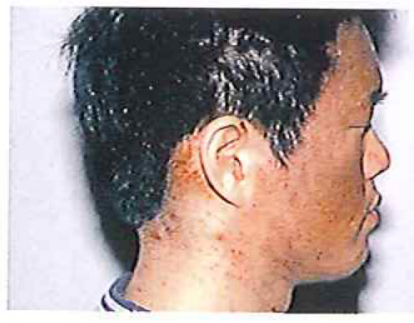

b

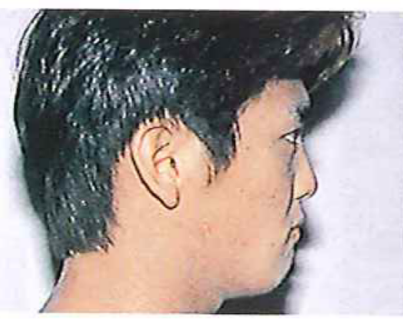

c

d

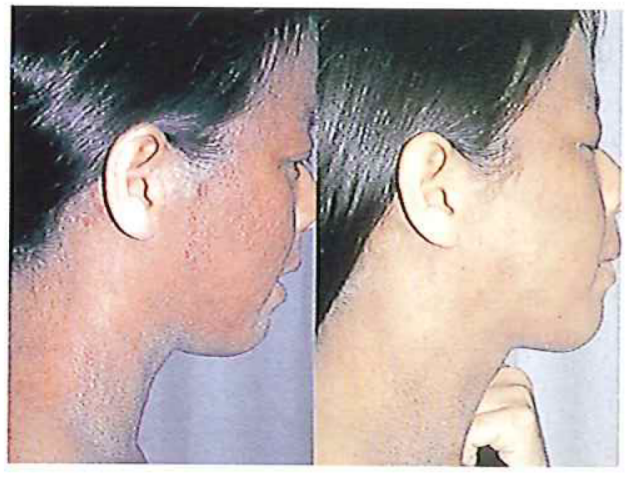

k

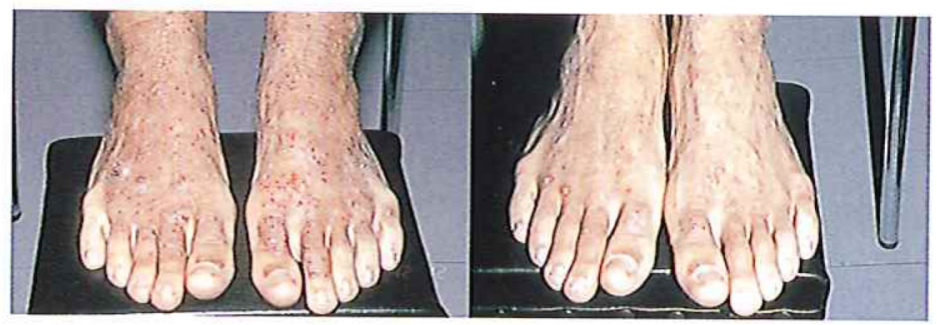

e f

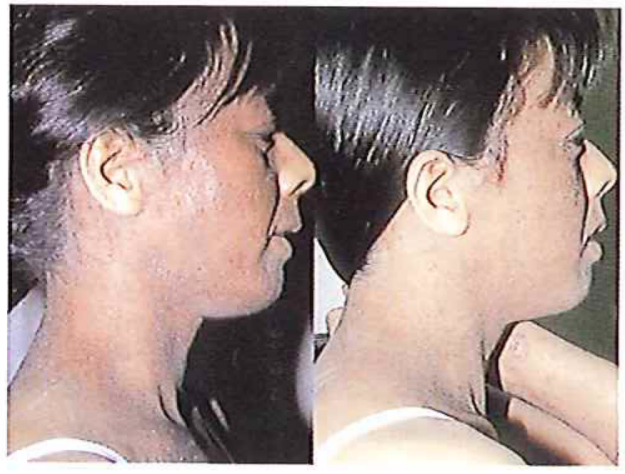

g

h

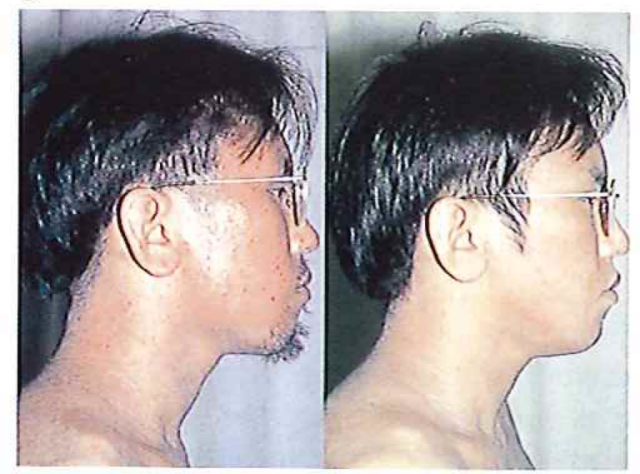

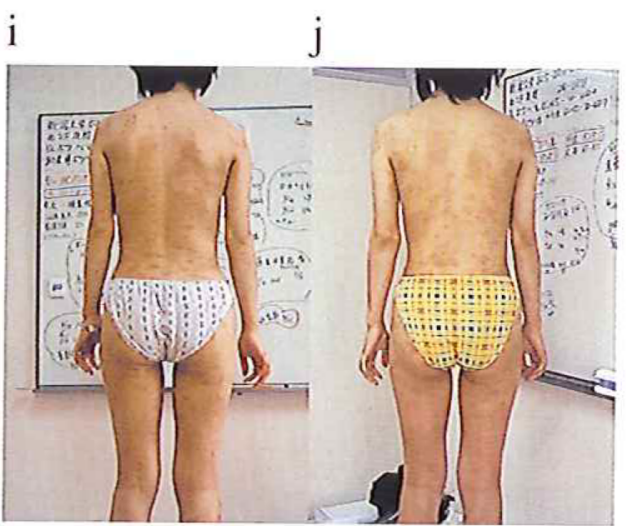

m

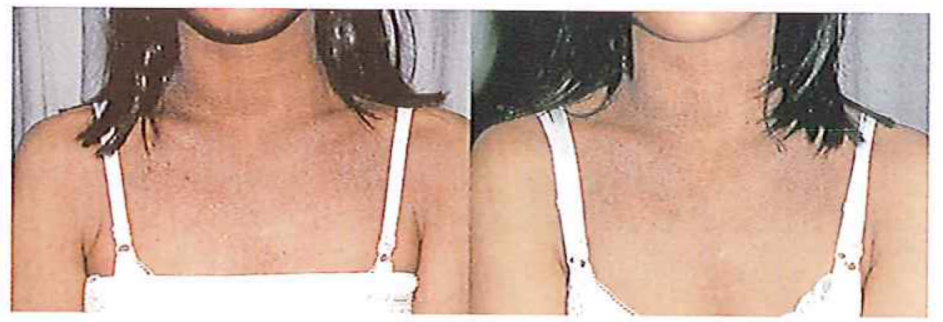

Fig. 3 Successful treatment of severe atopic dermatitis by acupuncture. Right: before treatment (a, c, e, g, i, k, m, and $\mathrm{o}$ ), Left: after treatment $(\mathrm{a} \rightarrow \mathrm{b}, \mathrm{c} \rightarrow \mathrm{d}, \mathrm{e} \rightarrow \mathrm{f}, \mathrm{g} \rightarrow \mathrm{h}, \mathrm{i} \rightarrow \mathrm{j}, \mathrm{k} \rightarrow \mathrm{l}, \mathrm{m} \rightarrow \mathrm{n}$, and $\mathrm{o} \rightarrow \mathrm{p})$. After cessation of the use of ointment containing steroid hormones, the patients suffered from the withdrawal syndrome. This was, however, countered by acupuncture, resulting in successful treatment within one month to several months. 
Possible metabolism of administered steroids

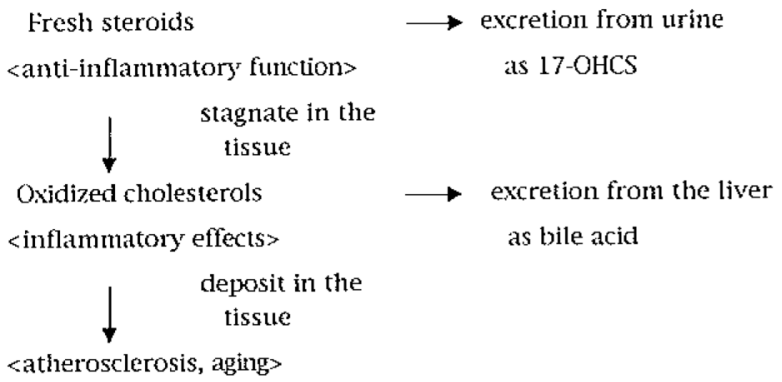

Fig. 4 Fate of administered steroids in patients with atopic dermatitis

from circulation failure or other ailments (our unpublished observations).

As shown by the present data, many of these patients were genetically predisposed to atopy as reflected by the high level of eosinophils before and after therapy. In this regard, some patients suffered a recurrence of disease. However, possibly because of their ages (older than 16 years), the recurrence ratio was not so high (less than $10 \%$ ). In the case of patients with atopic dermatitis who had not yet used ointment containing steroid hormones, two or three weeks were sufficient to achieve successful treatment by acupuncture. In other words, severe cases of atopic dematitis might result from long use of steroid hormones.

With the present concept, we can easily understand the reason for the difficulty in reducing doses of steroid hormones in many diseased persons who have used such hormones for a long time. We call it steroid hormone addiction. Increased amounts of steroid hormones are always required for neutralization (but still temporary) of the stagnated, oxidized substance.

Our hypothesis still remains a speculation, and we also have to consider many other reasons. Not only $\mathrm{T}$ cells, IgE, and eosinophils but also granulocytes might be highly associated with severity of atopic dermatitis. Thus, granulocytosis was always accompanied with severe cases of atopic dermatitis and with the withdrawal syndrome. Although we do not yet know the precise mechanisms underlying granulocytosis, one of the candidates may be stagnated steroid hormones which then induce sympathetic nerve activation.

\section{Note}

Subsequent to collection of the data on the patients herein reported, we achieved successful treatment of 500 other patients with severe atopic dermatitis.

\section{REFERENCES}

1. Bos J. D., Van Leent E. J. M. and Smitt J. H. S. (1998) The millennium criteria for the diagnosis of atopic dermatitis. Exp. Dermatol. 7, 132-138.

2. Finlay A. Y. (1996) Measurement of disease activity and outcome in atopic dermatitis. Br. J. Dermatol. 135, 509-515.

3. Kaplan R. J. and Rosenberg E. W. (1978) Atopic dermatitis: clinical and immunologic aspects and treatment. Postgrad. Med. 64, 52-56.

4. Kawamura H., Kawamura T., Kokai Y., Mori M., Matsuura A., Oya H., Honda S., Suzuki S., Weerasinghe A., Watanabe H. and Abo T. (1999) Expansion of extrathymic T cells as well as granulocytes in the liver and other organs of G-CSF transgenic mice. Why they lost the ability of hybrid resistance. J. Immunol. 162, 5957-5964.

5. Kawamura T., Toyabe S., Moroda T., Iiai T., TakahashiIwanaga H., Fukuda M., Watanabe H., Sekikawa H., Seki S. and Abo T. (1997) Neonatal granulocytosis is a postpartum event which is seen in the liver as well as in the blood. Hepatology, 26, 1567-1572.

6. Lesko M. J., Lever R. S., Mackie R. M. and Parrott D. M. (1989) The effect of topical steroid application on natural killer cell activity. Clin. Exp. Allergy 19, 633-636.

7. Maruyama S., Minagawa M., Shimizu T., Oya H., Yamamoto S., Musha N., Abo W., Weerasinghe A., Hatakeyama K. and Abo T. (1999) Administration of glucocorticoids markedly increases the numbers of granulocytes and extrathymic $\mathrm{T}$ cells in the bone marrow. Cell. Immutmol. 194, 28-35.

8. Minagawa M., Narita J., Tada T., Maruyama S., Shimizu T., Bannai M., Oya H., Hatakeyama K. and Abo T. (1999) Mechanisms underlying immunologic states during pregnancy: possible association of the sympahtetic nervous system. Cell. Immunol. 196, 1-13.

9. Miyaji C., Watanabe H., Minagawa M., Toma H., Nohara Y., Nozaki H., Sato Y. and Abo T. (1997) Numerical and functional characteristics of lymphocyte subsets in centenarians. J. Clin. Immunol. 17, 420-429.

10. Moroda T., Iiai T., Tsukahara A., Fukuda M., Suzuki S., Tada T., Hatakeyama K. and Abo T. (1997) Association of granulocytes with ulcer formation in the stomach of rodents exposed to restraint stress. Biomed. Res. 18, 423-437.

11. Nishijo K., Mori H., Yoshikawa K. and Yazawa K. (1997) Decreased heart rate by acupuncture stimulation in humans via facilitation of cardiac vagal activity and suppression of cardiac sympathetic nerve. Neurosci. Lett. 227, 165-168.

12. Rajka G. (1986) Natural history and clinical manifestations of atopic dermatitis. Clin. Rev. Allergy' 4, 3-26.

13. Rajka G. and Langeland T. (1989) Grading of the severity of atopic dermatitis. Acta Derm. Venereol. 144, 13-14.

14. Rogge J. L. and Hanifin J. M. (1976) Immunodeficiencies in severe atopic dermatitis. Arch. Dermatol. 112, 1391-1396.

15. Suzuki S., Toyabe S., Moroda T., Tada T., Tsukahara A., Iiai T., Minagawa M., Maruyama S., Hatakeyama K., Endo K. and Abo T. (1997) Circadian rhythm of leukocytes and lymphocyte subsets and its possible correlation with the function 
of autonomic nervous system. Clin. Exp. Immumol. 110, 500508.

16. Tam K. C. and Yiu H. H. (1975) The effect of acupuncture on essential hypertension. Am. J. Chin. Med. 3, 369-375.

17. Toyabe S., liai T., Fukuda M., Kawamura T., Suzuki S., Uchiyama M. and Abo T. (1997) Identification of nicotinic acetylcholine receptors on lymphocytes in periphery as well as thymus in mice. Immmology 92, 201-205.

18. Vandercam B., Lachapelle J. M., Janssens P., Tennstedt D. and Lambert M. (1997) Kaposi's sarcoma during immunosuppressive therapy for atopic dermatitis. Dermatology 194, 180182.

19. Walker C., Kagi M. K., Ingold P., Braun P., Blaser K., Bruijnzeel-Koomen C. A. and Wuthrich B. (1993) Atopic dermatitis: correlation of peripheral blood $\mathrm{T}$ cell activation, eosi- nophilia and serum factors with conical severity. Clin. Exp. Allergy, 23, 145-153.

20. Werfel T. and Kapp A. (1999) What do we know about the etiopathology of the intrinsic type of atopic dermatitis? Cur: Probl. Dermatol. 28, 29-36.

21. Williams T., Mueller K. and Cornwall M. W. (1991) Effect of acupuncture-point stimulation on diastolic blood pressure in hypertensive subjects: a preliminary study. Phys. Ther: 71, 523-529.

22. Yamamura S., Arai K., Toyabe S., Takahashi E. H. and Abo T. (1996) Simultaneous activation of granulocytes and extrathymic $T$ cells in number and function by excessive administration of nonsteroidal anti-inflammatory drugs. Cell. Immunol. 173, 303-311. 